\title{
Labeling the Brain Surface Using a Deformable Multiresolution Mesh
}

\author{
Sylvain Jaume ${ }^{1}$, Benoît Macq ${ }^{2}$, and Simon K. Warfield ${ }^{3}$ \\ 1 Multiresolution Modeling Group, California Institute of Technology, 256-80, \\ 1200 E. California Blvd, Pasadena, CA 91125, USA, jaume@caltech.edu \\ 2 Telecommunications and Remote Sensing Lab, Université catholique de Louvain, \\ Place du Levant, 2, 1348 Louvain-la-Neuve, Belgium \\ 3 Surgical Planning Laboratory, Brigham and Women's Hospital, \\ Harvard Medical School, 75 Francis St, Boston, MA 02115, USA
}

\begin{abstract}
We propose to match a labeled mesh onto the patient brain surface in a multiresolution way for labeling the patient brain. Labeling the patient brain surface provides a map of the brain folds where the neuroradiologist and the neurosurgeon can easily track the features of interest. Due to the complexity of the cortical surface, this task usually depends on the intervention of an expert, and is time-consuming. Our multiresolution representation for the brain surface allows the automated classification of the folds based on their size. The atlas mesh is deformed from coarse to fine to robustly capture the patient brain folds from the largest to the smallest. Once the atlas mesh matches the patient mesh, the atlas labels are transferred to the patient mesh, and color coded for visualization.
\end{abstract}

Keywords: cortex labeling, cortical surface, sulci and gyri, brain segmentation, brain atlas, multiresolution mesh, progressive mesh.

\section{Introduction}

To diagnose a neural disorder or to preserve the main functions of the patient during a brain operation, the neuroradiologist and the neurosurgeon need to locate regions of interest relative to the folds (sulci and gyri) on the patient brain. Some software have been developed to ease the labeling of sulci in the patient brain scan. Since the brain geometry exhibits a high complexity and a high inter-patient variability, the labeling software usually requires the input from an expert. Unfortunately the required manual intervention makes the labeling both time-consuming and operator-dependent. Automated labeling of the sulci and gyri of the brain would be a significant aid in studying brain structure and function, with clinical applications ranging from surgical planning to the study of structural changes associated with brain disorders.

First we review some methods for automating the labeling of the cortex. At the end of this section, we briefly present our contribution and the outline of the paper. 


\subsection{Related Work}

Sandor et al. 1] elastically deform a reference labeled brain volume (brain atlas) to fit the patient brain scan. Mathematical morphology and edge detection operations convert the patient scan to a smooth representation the deformable surface can easily match. The brain atlas is parameterized with B-spline surfaces and is deformed under an energy minimization scheme. The energy attracts the atlas fold points to fold points on the patient image, and the remaining points to the patient brain surface. Finally atlas labels are transfered to the patient brain surface. However, due to the low resolution in regions of the brain scan (partial volume effect), the morphological processing can accidentally disconnect or erase brain folds on the patient image. Consequently the deformable surface might be unable to capture the disconnected brain folds.

Lancaster et al. 2] use axis aligned coordinates to retrieve brain labels from the Tailarach atlas. They hierarchically subdivide the large atlas structures into substructures. For instance, the cerebrum is subdivided into lobes and sublobar structures, which are then subdivided into smaller structures. The Tailarach coordinates are addressed in a volumetric image synthesized from the Tailarach atlas. Some rules guide the segmentation and labeling of 160 contiguous regions. They compare their automated labeling with manual labeling [3] and show $70 \%$ or greater label match on 250 functional MRI scans.

Le Goualher et al. 4] extract surface ribbons that represent the median axis of the sulci for interactive sulci labeling. They build a graph where the nodes define the sulci with some geometric and anatomical attributes, and the arcs define the connections between the sulci. For every node, the expected spatial distribution of the sulci is computed and the most likely labels are returned to the operator. Then the operator needs to pick the correct label and associate it to the node. This scheme eases the task of the expert, but still requires some intervention from the user.

Lohmann et al. 5] segments the patient brain scan with a region growing method. The segmented regions, called sulcal basins, represent substructures of the brain folds. A point distribution model is used to match an atlas model onto the patient brain, and to label the sulcal basins. Since the geometric difference between the atlas and the patient brain can be high, the point distribution model can accidentally match an atlas sulcus to the wrong sulcus on the patient anatomy.

Hellier et al. 6] extract the sulci with an active ribbon method. Using a nonrigid registration, brain images from different subjects are matched to minimize the sum of the distances between corresponding sulci. The authors demonstrate that their scheme accurately matches six brain labels. However, it was not shown that small sulci can robustly be segmented and labeled with their non-rigid registration method.

Rivière et al. 7] use a graph representation of the brain folds similar to Le Gouahler and match a graph with labels to the graph of the patient brain. For every brain fold, i.e. a node in the graph, the likelihood of assigning a particular label is computed from geometric measures and the connectivity to neighboring 


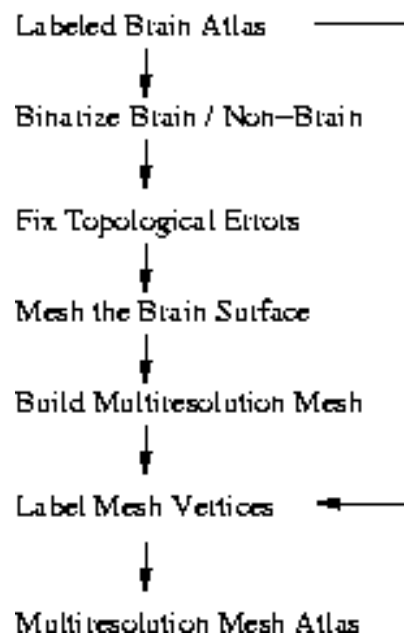

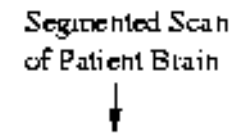

Build Multilesolution

Mers<smiles>[3H][V]</smiles>

Loxale Sulti

at + Resolutions

$\checkmark$

Mateh Sulci ftoun At las Mers to Patient Merh

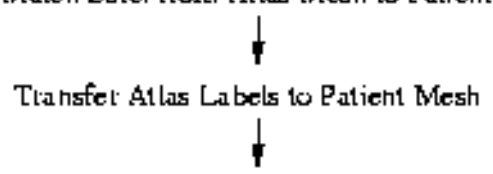

Labeled Patient Mesh

Fig. 1. We build a multiresolution mesh from a labeled brain scan (brain atlas) (left). We build a similar structure for the patient mesh and detect the folds at 4 resolutions for both meshes. Then we deform the labeled mesh onto the patient mesh to match folds of similar sizes. Finally we transfer the labels to the patient mesh. (right)

folds. Then a matching method based on neural networks minimizes a combination of the likelihoods.

Cachier et al. [8] show that combining the previous method with feature point matching improves the registration of brain scans. They register differently the bottom line of the sulci from the upper line of the gyri, since the localization of the latter exhibits higher inter-subject variability.

\subsection{Our Contribution}

We propose to progressively match an atlas labeled mesh to the patient brain mesh from the largest folds to the smallest folds. Then we transfer the labels from the matched mesh to label the patient mesh without manual intervention.

\section{Algorithm}

First we build a multiresolution mesh of the brain surface from a reference labeled brain scan (atlas). Every mesh vertex is assigned the closest label in the atlas. Then we apply some geometric operations to the multiresolution mesh to extract and classify the brain folds according to their size. Finally we match the labeled mesh onto the patient mesh and transfer the labels to the patient anatomy. To extract a surface mesh from the patient brain scan, brain tissues and non-brain tissues must be classified before the application of our algorithm. Fig 1 illustrates the steps to build a multiresolution mesh from a segmented brain scan on the left diagram. The right diagram shows the automated labeling of a patient brain mesh. 


\subsection{Building the Multiresolution Mesh}

We create a mesh of the brain surface from a labeled atlas volume [9]. To extract a surface from the volume, we set all brain labels to white and the remaining to black. However 366 tiny holes and handles in the binary volume prevents to extract a brain surface with a spherical topology. These topological errors are artifacts of the magnetic resonance image (MRI) (partial volume effect) or local voxel misclassifications. We thus apply the automated method of Wood et al. [10] to remove the holes and handles with minimal modification to the binary volume. Then we generate a triangle mesh of the brain surface with the Marching Cubes [11. Finally we associate to every mesh vertex the label of the closest structure in the atlas. The brain mesh with 35 labels is shown in Fig 2 (left).

Now we build a multiresolution representation of the brain mesh to enable geometric manipulations. We use the Progressive Mesh structure that Hoppe [12] introduced as a sequence of vertices that progressively refines a coarse mesh. Fig 2 shows a coarse brain mesh and a refined mesh in the center left and center right images respectively. Combining the mesh hierarchy of Guskov et al. 13. and the local operator of Desbrun et al. [14, we encode for every vertex in the sequence the difference its insertion makes to the local mesh geometry. We can perform various geometric operations on the brain surface, such as smoothing and matching.

The first three images in Fig 2 shows that the multiresolution labeled mesh can be represented at various resolutions. The fine geometric information is encoded based on larger structures. This hierarchical representation will help to match the labeled mesh onto a patient brain mesh, such as the mesh shown in the fourth image.

\subsection{Extracting the Sulci}

We smooth the brain surface at four resolutions. The regions where the geometry significantly changes from the previous resolution define brain folds with similar sizes. We thus classify the brain folds into four size categories. Fig 3 show the brain folds classification for the reference mesh in the first row, and for a patient brain mesh in the second row. The largest folds (light blue) appear at the smoothest resolution, and progressively smaller folds (green, yellow ang red respectively), appear on the successive representations. The regions without significant geometric difference between two representations the remaining brain surface (dark blue).

\subsection{Matching the Labeled Mesh onto the Patient Mesh}

We take advantage of the multiresolution mesh representation and the classification of the brain folds to constrain the matching from the atlas mesh to the target mesh. A coarse to fine matching first allows large surface deformations and then more subtle deformations. We also use our fold classification as a constraint: large folds on the atlas must match large folds on the patient brain, and 
similarly for the small folds. We repeatedly move every vertex of the deformable mesh along its local normal to the closest intersection with the surface of the patient mesh. The last row of Fig 3 illustrates the multiresolution matching of the labeled mesh (first row) onto the patient mesh (second row). From left to right, the matching evolves from a smooth representation to the original representation. First the smoothest representations are registered to match the center fold (light blue). As smaller brain folds are introduced on the successive representations, they are matched onto the patient surface (green, yellow, and red). The final match captures the brain folds on the original patient mesh.

\section{Results and Discussion}

We matched the labeled mesh for nine cases with a brain tumor. The tumor deforms the patient brain anatomy. The pathological deformation adds to the inter-subject variability and makes the matching of the atlas mesh to the patient mesh even more difficult. After atlas matching, we transfer and color code the atlas labels onto the patient mesh. Fig 4 shows the nine patient meshes after labeling. Visualization of the labels indicates that the multiresolution matching accurately captures the patient brain folds despite the high difference from the atlas geometry. It took less than 8 minutes on a Pentium 4 2GHz to automatically label a new patient scan. This makes the software suitable as a clinical aid. Future validations will compare our automated labeling with manually labeled data, quantify the accuracy of our method.

\section{Conclusion}

We match the labeled surface of a brain atlas to the patient anatomy from the largest folds to the smallest folds. A multiresolution mesh built for every brain scan allows the classification of the folds based on their size. To robustly match the atlas mesh, we first match the largest folds, and progressively match the smaller folds. Once the atlas mesh fits the smallest patient folds, the atlas labels are transfered to the patient mesh. The multiresolution matching provides the neurologist with a map of the patient brain in a short time and with no intervention. We ran our method to automatically label nine brain scans with a significant pathological deformation. Visualization of the labeled patient meshes indicates that the labels closely match the folds on the patient anatomy.

Acknowledgements: Special thanks to Zoë Wood for running her algorithm on every case, Peter Schröder and Mathieu Desbrun for insightful discussions, and Christopher Malek for making this research possible. Thanks to Ron Kikinis, Martha Shenton, Robert McCarley, and Ferenc Jolesz for sharing the atlas volume. Sylvain Jaume is working towards a Ph.D. degree with awards from FRIA and FNRS (Belgian Science Foundation). This investigation was supported by NIH grants P41 RR13218, P01 CA67165, R01 RR11747, R01 CA86879, by a research grant from the Whitaker Foundation, and by a New Concept Award from the Center for Integration of Medicine and Innovative Technology. 

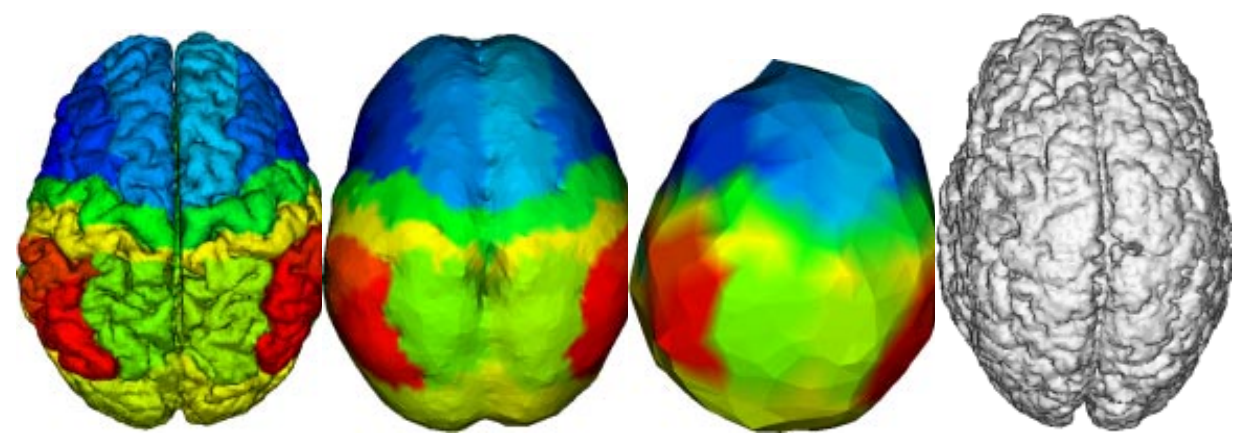

Fig. 2. We label a surface mesh from a labeled brain scan (left) and represent it in multiresolution (center left and center right). The multiresolution representation allows the matching of the labeled mesh onto a patient brain mesh (right).

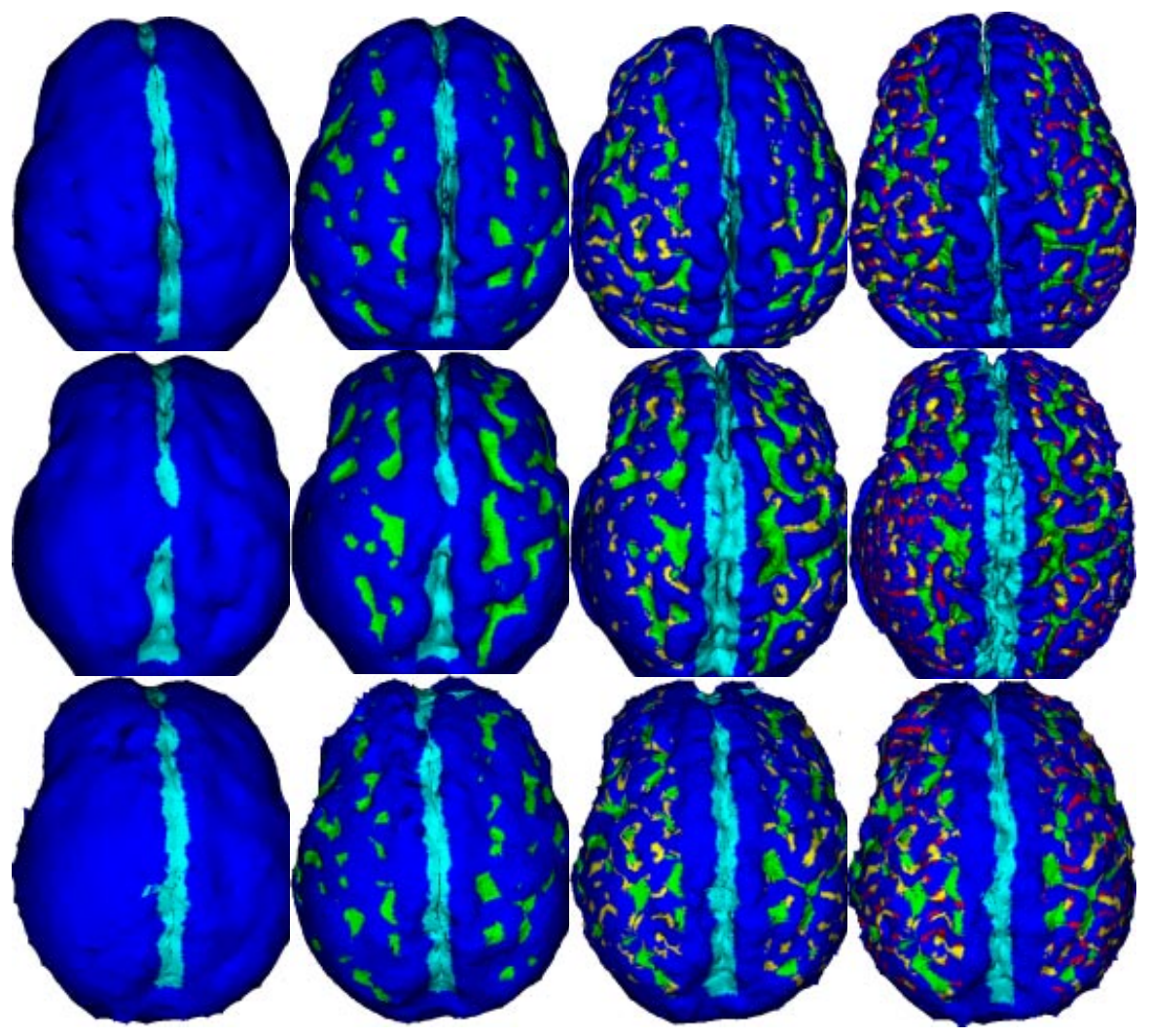

Fig. 3. The multiresolution representation of the cortex allows to distinguish the large sulci from the the small sulci. The first structure to appear is the fold between the hemispheres (light blue). The remaining brain surface is colored in dark blue. Then the sulci appear from the largest to the smallest (respectively green, yellow, and red). In the second row, we perform the sulci classification on a mesh of the patient brain. In the third row, the multiresolution mesh is progressively deformed to match the corresponding folds on the patient mesh. 


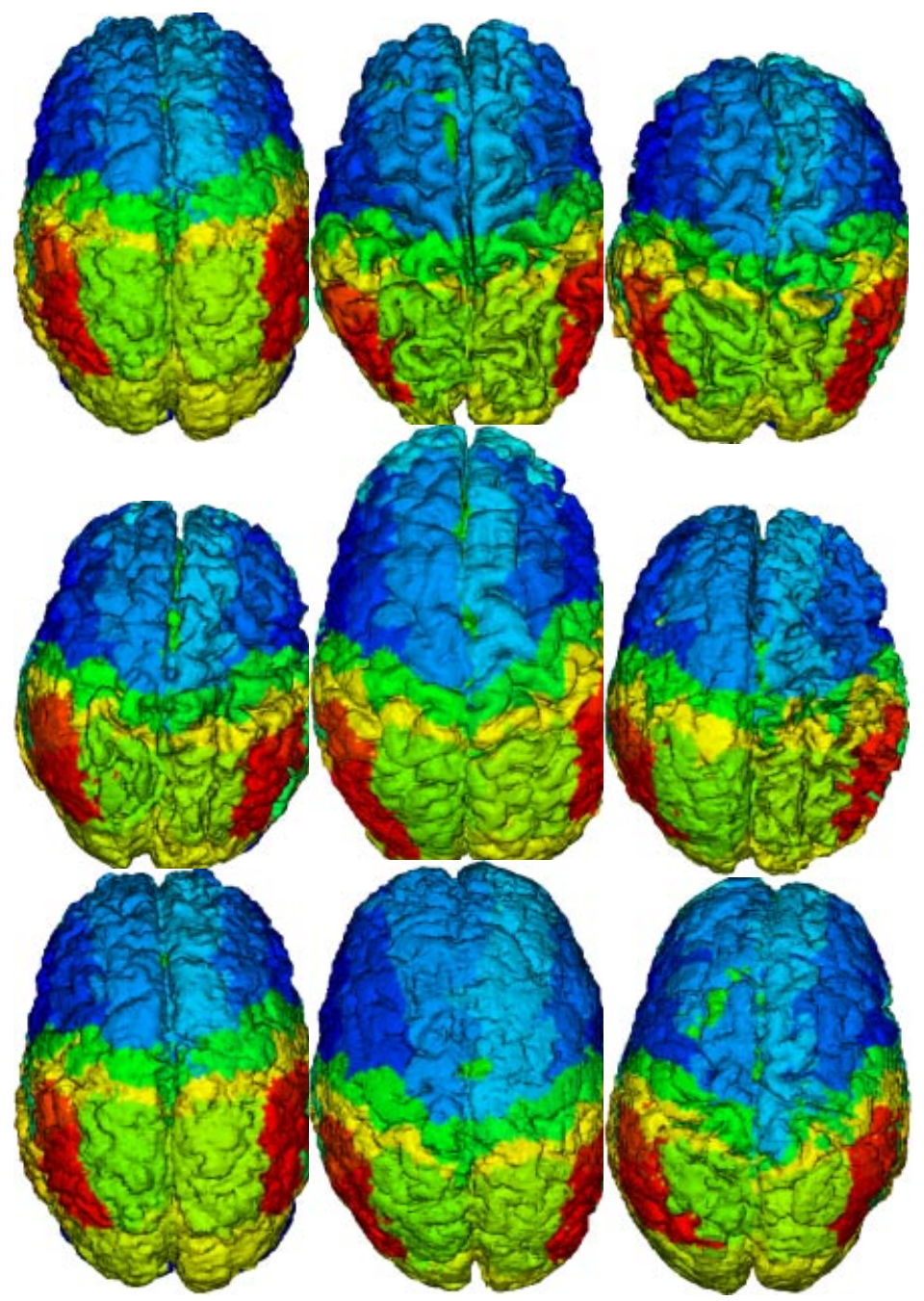

Fig. 4. We transfer the 35 atlas labels to the patient mesh after matching the atlas mesh onto nine cases. The nine cases were thus labeled without manual intervention. The visualization of the labels with color coding indicates that the labels accurately match the patient brain geometry.

\section{References}

1. S. Sandor and R. Leahy. Surface-based labeling of cortical anatomy using a deformable atlas. IEEE Transactions on Medical Imaging, 16(1):41-54, February 1997.

2. J.L. Lancaster, L.H. Rainey, J.L. Summerlin, C.S. Freitas, P.T. Fox, A.C. Evans, A.W. Toga, and J.C. Mazziotta. Automated labeling of the human brain: a preliminary report on the development and evaluation of a forward-transform method. Human Brain Mapping, 5(4):238-242, 1997. 
3. J.L. Lancaster, M.G. Woldorff, L.M. Parsons, M. Liotti, C.S. Freitas, L. Rainey, P.V. Kochunov, D. Nickerson, S.A. Mikiten, and Fox P.T. Automated tailarach atlas labels for functional brain mapping. Human Brain Mapping, 10(3):120-131, July 2000.

4. G. Le Goualher, E. Procyk, D.L. Collins, R. Venugopal, G., C. Barillot, and A.C. Evans. Automated extraction and variability analysis of sulcal neuroanatomy. IEEE Transactions on Medical Imaging, 18(3):206-217, March 1999.

5. G. Lohmann and D.Y. von Cramon. Automatic labelling of the human cortical surface using sulcal basins. Medical Image Analysis, 4(3):179-188, September 2000.

6. P. Hellier and Barillot C. Coupling dense and landmark-based approaches for non rigid registration. Technical report, Institut de Recherche en Informatique et Systèmes Aléatoires (IRISA), November 2000.

7. D. Rivière, J.-F. Mangin, D. Papadopoulos, J.-M. Martinez, V. Frouin, and J. Régis. Automatic recognition of cortical sulci unisng a congregation of neural networks. In Third International Conference on Medical Robotics, Imaging and Computer Assisted Surgery - MICCAI 2000, pages 40-49, Pittsburgh, USA, October 11-14 2000.

8. P. Cachier, J.-F. Mangin, X. Pennec, D. Rivière, D. Papadopoulos-Orfanos, J. Régis, and N. Ayache. Multisubject non-rigid registration of brain mri using intensity and geometric features. In Fourth International Conference on Medical Image Computing and Computer-Assisted Intervention - MICCAI 2001, volume 2208, pages 734-742, Utrecht, The Netherlands, October 14-17 2001.

9. Kikinis et al. A digital brain atlas for surgical planning model driven segmentation and teaching. IEEE Transactions on Visualization and Computer Graphics, 2(3), September 1996.

10. Z. Wood, H. Hoppe, M. Desbrun, and P. Schröder. Isosurface topology simplification. ACM Transactions on Graphics, 2002. submitted.

11. W.E. Lorensen and H.E. Cline. Marching cubes: a high resolution 3D surface construction algorithm. In Proceedings of Computer Graphics SIGGRAPH, pages 163-169, Anaheim, USA, July 1987.

12. H. Hoppe. Progressive meshes. In Proceedings of Computer Graphics SIGGRAPH, New Orleans, USA, August 4-9 1996.

13. I. Guskov, W. Sweldens, and P. Schröder. Multiresolution signal processing for meshes. In Proceedings of Computer Graphics SIGGRAPH, Los Angeles, USA, August 8-13 1999.

14. M. Desbrun, M. Meyer, P. Schröder, and A. Barr. Implicit fairing of irregular meshes using diffusion and curvature flow. In Proceedings of Computer Graphics SIGGRAPH, Los Angeles, USA, August 8-13 1999. 